\title{
基于能量衰减法利用CHAMP卫星精密轨道数据 反演热层大气密度
}

\author{
李若曦 ${ }^{1}$, 雷久侯 ${ }^{1 *}$, 王西京 ${ }^{2}$, 窦贤康 ${ }^{1}$, 金双根 ${ }^{3}$ \\ 1. 中国科学技术大学地球和空间科学学院, 合肥 230026 ; \\ 2. 航天器在轨故障诊断与维修重点实验室, 西安 710043; \\ 3. 中国科学院上海天文台, 上海 200030 \\ *通讯作者, E-mail: leijh@ustc.edu.cn \\ 收稿日期: 2017-03-04; 接受日期: 2017-05-10; 网络版发表日期: 2017-07-11 \\ 国家自然科学基金项目(批准号: 41325017, 41274158)资助
}

摘要利用CHAMP卫星精密轨道数据, 使用新的反演方法, 即能量衰减法反演了热层大气密度, 并将反演结 果与加速度计数据反演的大气密度以及经验模式结果对比. 主要结论如下: (1) 利用卫星精密轨道数据, 可以使 用能量衰减法反演热层大气密度, 其反演结果与半长轴衰减法一致; (2) 反演密度的时间间隔与反演精度相关. 对于CHAMP卫星, 采用 $20 \mathrm{~min}$ 左右的积分时间较为合适; (3) 应用能量衰减法反演密度, 可同时对加速度计数据 进行标定; (4) 使用精密轨道数据反演密度精度与卫星高度、地方时等相关.

关键词热层大气, 密度, 卫星轨道, 半长轴, 能量, 加速度计

\section{1 引言}

热层大气对低轨航天器的拖曳作用直接影响着航 天器的控制以及寿命(Emmert, 2015). 目前在工程应用 中普遍采用DTM(Berger等, 1998)和MSIS(Hedin, 1987) 等经验模式计算卫星轨道动力学中的大气摄动力. 一 般情况下, 热层大气模式计算出的密度平均误差约为 15 20\%(Marcos, 1990), 且在地磁活动期间, 经验模式 计算出的大气密度误差很大(Emmert, 2015; Bowman 等, 2008). 因此, 热层大气密度的观测、反演以及预报 有着重要的应用意义.

Picone等(2005)采用北美防空司令部(NORAD)发
布的两行数据(TLE), 反演热层大气密度, 但是由于 两行数据密度低, 轨道数据点每天只有几个, 反演结 果时间分辨率低。随着 CHAMP(Reigber等, 2002)和 GRACE(Tapley等, 2004)等携带高精度加速度计的卫 星发射升空, 多个研究组(Bruinsma和Biancale, 2003; Sutton等, 2007; Doornbos, 2012; 李文文等, 2016)使用 星载加速度计数据实现了对热层大气密度高时空分辨 率的计算. 利用加速度计数据反演的热层大气密度结 果已广泛应用于热层大气密度对太阳风暴、地磁暴 等空间天气事件的响应等相关的研究中(Lei等, 2011a, 2012). 加速度计数据需要经过标定才可以使用, 很多 学者(Sutton等, 2007; Doornbos, 2012)采用直接定轨的

\footnotetext{
中文引用格式: 李若曦, 雷久侯, 王西京, 窦贤康, 金双根. 2017. 基于能量衰减法利用CHAMP卫星精密轨道数据反演热层大气密度. 中国科学: 地球科 学, 47: 1084-1096, doi: 10.1360/N072016-00373

英文引用格式： Li R X, Lei J H, Wang X J, Dou X K, Jin S G. 2017. Thermospheric mass density derived from CHAMP satellite precise orbit determination data based on energy balance method. Science China Earth Sciences, 60: 1495-1506, doi: 10.1007/s11430-016-9052-1
} 
方法估计标定系数. 熊永清等(2011)提出以CHAMP卫 星 RSO数据作为伪观测量, 以带参数的加速度计数据 替换非保守力模型再次定轨的方法估计加速度标定 因子, 能够降低使用GPS观测量直接定轨的复杂程度. 徐天河和杨元喜(2004)使用能量守恒的方法, 在现有 高精度地球重力场的基础上, 也可以较为准确的估计 出标定因子, 而且运算更为高效. 只有少数卫星携带 高精度加速度计, 使用加速度计数据反演大气密度的 方法受制于探测仪器数量. 因此, Calabia和 Jin(2016) 使用GRACE卫星精密轨道数据, 通过差值的方法反 演了热层大气密度, 但这种方法的正确性严重依赖于 GRACE卫星精密轨道数据较高的数据密度; Sang 等 (2012)使用CHAMP卫星精密轨道数据, 反演得到高时 空分辨的热层大气密度, 但该工作仅计算了几天结果, 没有长时间统计分析比较, 而且没有考虑时变的卫星 姿态以及阻尼系数所造成的影响.

本文提出一种新的热层大气密度反演方法, 即利 用大气拖曳力做功造成的能量的衰减反演热层大气 密度. 这种方法由于不需要数值积分, 可以大幅提升 运算效率, 并且该反演过程可同时对CHAMP卫星加 速度计数据进行标定. 最后本文将精密轨道数据反 演密度与加速度计数据反演结果及MSIS00(Picone等, 2002)结果进行对比验证与统计分析, 并分析精密轨道 数据反演密度方法的适用条件.

\section{2 大气密度反演方法介绍}

\section{1 半长轴衰减法反演热层大气密度}

根据人造地球卫星摄动方程(刘林, 1992), 卫星半 长轴变化率为

$$
\frac{\mathrm{d} a}{\mathrm{~d} t}=\frac{2}{n \sqrt{1-e^{2}}}\left(1+2 e \cos f+e^{2}\right)^{1 / 2} U,
$$

式中, $a 、 e 、 f$ 和 $n$ 分别代表半长轴、偏心率、真近点 角和平运动角速度, $U$ 代表切向摄动力. 这里切向摄动 力即大气拖曳力, 可以表示为

$$
U=\frac{1}{2} \frac{C_{D} A}{m} \rho v_{r}^{2},
$$

式中, $C_{D}$ 代表阻尼系数, $A$ 代表有效横截面积, $m$ 代表 卫星质量, $\rho$ 代表大气密度, $v_{r}$ 代表卫星相对大气运动 速度, 这里认为大气与地球具有相同自转角速度. 因
此根据式(1)和(2), 大气密度可以表示为

$$
\rho=-\frac{\mathrm{d} a}{\mathrm{~d} t} /\left(\frac{C_{D} A}{m} v_{r}^{2} \frac{\left(1+2 e \cos f+e^{2}\right)^{1 / 2}}{n \sqrt{1-e^{2}}}\right) .
$$

本文所采用的数据是CHAMP卫星快速科学轨道 数据(RSO). CHAMP卫星是德国于 2000 年 7 月发射升 空的一颗低轨卫星, 轨道近圆形, 倾角约 $87^{\circ}$, 其主要 的科学目的是测量地球重力场, 由亥姆霍兹波茨坦中 心(GFZ)管理并发布数据(Reigber等, 2002). RSO数据 是GFZ发布的第三阶(Level3)轨道(Orbit/Gravity)数据 中的一种, 通过GPS定轨, 每30s给出一组卫星状态量 (位置、速度)(König等, 2001). 其定轨精度在发射初 期约为 $20 \mathrm{~cm}$, 后期稳定运行期间约为 $5 \mathrm{~cm}$ (Michalak等, 2003). 以某一时刻 $t_{0}$ 作为初始状态量, 经过某一时间 间隔 $\Delta t$, 使用除大气拖曳力以外的所有摄动力进行轨 道外推, 可以计算出 $t_{1}$ 时刻卫星状态量, 这里 $t_{1}=t_{0}+\Delta t$,

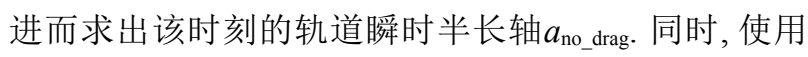
$t_{1}$ 时刻 RSO数据给出的卫星状态量, 计算该时刻轨道 真实瞬时半长轴 $a_{\text {real }}$, 因此半长轴由于大气拖曳力而造 成的衰减率 $\left.\frac{\mathrm{d} a}{\mathrm{~d} t}\right|_{\mathrm{drag}}$ 可以表示为(Sang 等, 2012)

$$
\left.\frac{\mathrm{d} a}{\mathrm{~d} t}\right|_{\text {drag }}=\frac{a_{\text {real }}-a_{\text {no_drag }}}{\Delta t},
$$

式中, $a 、 e 、 f$ 和 $n$ 等开普勒轨道根数均可由 $\mathrm{RSO}$ 数据 计算得到. 热层大气密度可由式(3)和(4)得到

$$
\rho=\frac{\left.\frac{\mathrm{d} a}{\mathrm{~d} t}\right|_{\mathrm{drag}}}{\left(\frac{C_{D} A}{m} v_{r}^{2} \frac{\left(1+2 e \cos f+e^{2}\right)^{1 / 2}}{n \sqrt{1-e^{2}}}\right)} .
$$

下面计算定轨误差对半长轴衰减量 $\Delta a=a_{\text {real }}-a_{\text {no _drag }}$ 的传递误差, 并分析定轨误差对密度反演的影响. 由 活力公式

$$
a=\left(\frac{2}{r}-\frac{v^{2}}{\mu}\right)^{-1},
$$

再根据测量误差传递公式, 可以计算半长轴的测量误 差为

$$
\delta_{a}^{2}=\frac{4 a^{4}}{r^{4}} \delta_{r}^{2}+\frac{4 v^{2} a^{4}}{\mu^{2}} \delta_{v}^{2} .
$$


这里做近似处理, 认为 $a \approx r=6750000 \mathrm{~m}, v=7500 \mathrm{~m} \mathrm{~s}^{-1}$, $\mu=3.986 \times 10^{14} \mathrm{~m}^{3} \mathrm{~s}^{-2}$, 位置与速度的测量精度约为: $\delta_{r}=0.05 \mathrm{~m}, \delta_{v}=0.00005 \mathrm{~m} \mathrm{~s}^{-1}$. 所以, 半长轴的测量误差 为 $\delta_{a}{ }^{2} \approx 4 \delta_{r}{ }^{2}+3 \times 10^{-6} \delta_{v}{ }^{2} \approx 0.13 \mathrm{~m}$.

这里认为 $a_{\mathrm{no} \_\mathrm{drag}}$ 与 $a_{\mathrm{real}}$ 具有相同的不确定度, 则 $\Delta a$ 的不确定度为

$$
\delta_{\Delta a}=\sqrt{2} \delta_{a}=0.18 \mathrm{~m} .
$$

定轨误差造成的 $\Delta a$ 的不确定度约为 $0.18 \mathrm{~m}$, 因此需要 选择合适的时间间隔 $\Delta t$, 使得在该时间间隔内半长轴 的衰减量至少大于 $0.18 \mathrm{~m}$.

\section{2 能量守恒法反演热层大气密度}

卫星在轨运动, 考虑EGM2008(Pavlis等, 2008)模 型100阶重力场, IERS2010(Petit和Luzum, 2010)给出的 固体潮重力场系数修正模型, 李济生(1995)给出的海 潮模型, 根据DE405星历所计算出的日月引力模型, 以 及大气拖曳力和光压等非保守力做功, 总能量可以表 示为

$$
\begin{aligned}
E_{0}= & \frac{1}{2}|\boldsymbol{v}|^{2}-V_{\mathrm{SUN}}-V_{\mathrm{MOON}}-\frac{1}{2} \omega^{2}\left(x^{2}-y^{2}\right) \\
& -V-E_{n},
\end{aligned}
$$

式中, $\frac{1}{2}|\boldsymbol{v}|^{2}$ 代表卫星动能, $V_{\mathrm{SUN}}$ 和 $V_{\mathrm{MOON}}$ 代表日月引力势 能, $\frac{1}{2} \omega^{2}\left(x^{2}-y^{2}\right)$ 代表旋转势能(Jekeli, 1999), $V$ 代表地 球重力场, $E_{n}$ 代表非保守力做功. 非保守力做功可以 表示为惯性系下, 非保守力在轨道路径上的积分, 即

$$
E_{n}=\int \boldsymbol{a} \cdot \boldsymbol{v} \mathrm{d} t,
$$

式中, $\boldsymbol{a}$ 代表非保守力产生的加速度, $\boldsymbol{v}$ 代表卫星速度. 以时刻 $t_{0}$ 作为初始状态量, 可以计算出该时刻卫星总 的机械能, 经过某一时间间隔 $\Delta t$ 计算出下一时刻 $t_{1}$ 的 卫星状态量, 进而求出 $t_{1}$ 时刻卫星机械能, 这里 $t_{1}=t_{0}+\Delta t$. 卫星在大气拖曳力和光压的作用下能量衰减, 能量的 衰减量就是两个时刻机械能的减少量, 即

$$
\begin{aligned}
& \int \boldsymbol{a}_{\mathrm{drag}} \cdot \boldsymbol{v} \mathrm{d} t+\int \boldsymbol{a}_{\text {solarpress }} \cdot \boldsymbol{v} \mathrm{d} t \\
= & \left(\frac{1}{2}|\boldsymbol{v}|^{2}-V_{\mathrm{SUN}}-V_{\text {MOON }}\right. \\
& \left.-\frac{1}{2} \omega^{2}\left(x^{2}-y^{2}\right)-V-E_{0}\right)\left.\right|_{t_{0}} ^{t_{1}},
\end{aligned}
$$

式中, $\boldsymbol{a}_{\mathrm{drag}} 、 \boldsymbol{a}_{\text {solarpress }}$ 分别代表大气拖曳力和太阳光压. 由于大气拖曳力沿卫星运动负方向, 大气拖曳力 做功可近似的写为

$$
\int \boldsymbol{a}_{\text {drag }} \cdot \boldsymbol{v} \mathrm{d} t=a_{\text {drag }}|\boldsymbol{v}| \Delta t .
$$

将式(2)所示大气拖曳力与式(11)所示非保守力做功带 入上式, $\Delta t$ 内卫星路径上的平均大气密度可以写为

$$
\begin{aligned}
\rho_{\text {drag }}= & \left(\frac{1}{2}|\boldsymbol{v}|^{2}-V_{\text {SUN }}-V_{\text {MOON }}-\frac{1}{2} \omega^{2}\left(x^{2}-y^{2}\right)\right. \\
& \left.-V-E_{0}-\int \boldsymbol{a}_{\text {solarpress }} \cdot \boldsymbol{v} \mathrm{d} t\right)\left.\right|_{t_{0}} ^{t_{1}} / \\
& \left(|\boldsymbol{v}| \Delta t \frac{1}{2} \frac{C_{D} A}{m} v_{r}^{2}\right) .
\end{aligned}
$$

下面分析半长轴衰减法与能量衰减法在反演大 气密度中的关系. 在极坐标系下, 卫星半长轴随时间 的变化方程如下(刘林, 1992):

$$
-\frac{G M}{a^{2}} \frac{\mathrm{d} a}{\mathrm{~d} t}=-2 \dot{\boldsymbol{r}} \cdot \boldsymbol{S}-2 r \dot{\boldsymbol{\theta}} \cdot \boldsymbol{T},
$$

式中, $r 、 \dot{\boldsymbol{r}} 、 \boldsymbol{\theta} 、 \dot{\boldsymbol{\theta}}$ 分别代表极坐标系下的位置、速 度、角度、角速度; $\boldsymbol{S} 、 \boldsymbol{T}$ 分别代表摄动力径向、横向分 量. 忽略摄动力径向分量, 式(14)与式(1)一致. 将式(14) 左边写为全微分形式, 并考虑到 $\dot{\boldsymbol{r}} \cdot \boldsymbol{S}+r \dot{\boldsymbol{\theta}} \cdot \boldsymbol{T}=\boldsymbol{F} \cdot \boldsymbol{V}$, 可以得到:

$$
\mathrm{d}\left(\frac{G M}{2 a}\right)=-\boldsymbol{F} \cdot \boldsymbol{V} \mathrm{d} t .
$$

由活力公式可知, $\frac{G M}{2 a}=\frac{G M}{r}-\frac{1}{2} v^{2}=E_{0}$, 所以式 (15)左边为能量的变化, 右边转化为非保守力做的功. 因此半长轴衰减法和能量衰减法二者数学原理上是 一致的, 区别在于半长轴衰减法需要数值积分, 这个 过程会引入误差. 本文中, 半长轴衰减法所使用到的 轨道外推时间较短, 数值积分过程误差很小.

\section{3 使用加速度计数据反演热层大气密度}

CHAMP卫星携带高精度加速度计, 加速度计沿卫 星运动方向具有十分高的测量精度, 精度可达 $3 \times 10^{-9} \mathrm{~m}$ $\mathrm{s}^{-2}$ (Flury等, 2008). 加速度计数据是GFZ发布的第二阶 (Level2)轨道(Orbit/Gravity)数据, 每 $10 \mathrm{~s}$ 一个数据点, 每 个数据点给出加速度初始测量值 $a_{0}$ 、洛伦兹修正项 $a_{L}$ 、径向修正项 $a_{t m}$ 以及姿态数据, 并记录卫星调姿时

1086 
刻(Förste等, 2002). 加速度计观测值 $a_{\text {observe }}$ 由下式给出:

$$
a_{\text {observe }}=a_{0}+a_{L}+a_{t m} .
$$

由于硬件问题, 加速度计观测值需要进一步标定, 标定满足(Bruinsma等, 2004):

$$
a_{I F X}=\text { Scale } \times a_{\text {observe }}+\text { Bias },
$$

式中, Scale和Bias分别代表尺度标定因子和偏差标定 因子. 加速度计数据经标定后为仪器坐标系下的观测 加速度, 用 $a_{I F X}$ 表示, 需要将其先变换到卫星坐标系下, 然后再变换到惯性坐标下才能使用(Lühr等, 2001):

$$
a_{I N F}=R_{I F X \rightarrow I N F} a_{I F X},
$$

式中, $R_{I F X \rightarrow I N F}$ 表示从仪器坐标系到惯性坐标系的坐标 变化矩阵. 最后, 根据式(2)计算大气密度:

$$
\rho=a_{I N F} /\left(\frac{1}{2} \frac{C_{D} A}{m} v_{r}^{2}\right) .
$$

将式(5)和(13)与上式对比可以发现, 使用精密轨道数 据反演密度实际上是先通过精密轨道数据数据计算 等效大气拖史加速度, 进而反演密度. 这里需要指出 的是, 加速度计测量的加速度为实时加速度, 精密轨 道数据计算的等效加速度是时间间隔 $\Delta t$ 内的平均加 速度, 其精度低于加速度计测量值.

加速度计数据标定方法以及结果将在 3.2 节中介 绍.

\section{3 有效面积、阻尼系数的计算以及加速度 计数据的标定}

\section{1 计算有效面积和阻尼系数}

卫星运行过程中质量基本保持不变, 由式(5)可 知, 要反演得到精确的大气密度, 必须精确计算阻尼 系数 $C_{D}$ 和卫星有效面积 $A$. 根据Sentman模型(Sentman, 1961), 卫星每一个面的阻尼系数和有效面积的乘积 $C_{D} \cdot A$ 可以表示为

$$
\left(C_{D} \cdot A\right)=\left(\frac{P}{\sqrt{\pi}}+\gamma Q Z+\frac{\gamma}{2} \frac{v_{\text {out }}}{v_{r}}(\gamma \sqrt{\pi} Z+P)\right) \cdot A,
$$

其中:

$$
\begin{aligned}
& G=\frac{1}{2 S^{2}}, P=\frac{1}{S} \exp \left(-\gamma^{2} S^{2}\right), \\
& Q=1+G, Z=1+\operatorname{erf}(\gamma S),
\end{aligned}
$$

$\gamma$ 代表卫星坐标系下, 某一卫星面板法向与 $-\boldsymbol{v}_{\boldsymbol{r}}$ 矢量方 向夹角的余弦函数, 即

$$
\gamma=-\boldsymbol{u}_{\boldsymbol{D}} \cdot \boldsymbol{u}_{N},
$$

式中, $\boldsymbol{u}_{N}$ 和 $\boldsymbol{u}_{\boldsymbol{D}}$ 分别代表面板法向和 $\boldsymbol{v}_{\boldsymbol{r}}$ 矢量的单位向量.

$S$ 代表卫星相对旋转大气速度和大气分子最慨然 热速度之比:

$$
S=\frac{v_{r}}{\sqrt{\frac{2 \mathrm{R} T}{m_{a}}}},
$$

式中, $T$ 和 $m_{a}$ 分别为使用MSIS 00 估算出的大气局地温 度和相对分子质量.

$T_{\text {in }}$ 代表入射大气粒子的均方根热力学温度:

$$
T_{\mathrm{in}}=\frac{m_{a} v_{r}^{2}}{3 \mathrm{R}},
$$

式中, $\mathrm{R}$ 代表热力学常数.

$v_{\text {out }}$ 代表出射大气粒子的速度:

$$
v_{\text {out }}=v_{r} \sqrt{\frac{2}{3}\left[1+\alpha_{E}\left(\frac{T_{\text {wall }}}{T_{\text {in }}}-1\right)\right]},
$$

式中, $T_{\text {wall }}$ 代表卫星表面温度. 这里认为卫星表面温度 很低, $T_{\mathrm{wall}}=300 \mathrm{~K} . \alpha_{E}$ 与大气相对分子质量和卫星材料 相对分子质量比相关, 可以表示为

$$
\alpha_{E}=\frac{2 \mu}{(1+\mu)^{2}},
$$

式中, $\mu$ 代表大气相对分子质量和卫星材料相对分子质 量之比. 这里考虑到卫星材料主要为氧硅材料, 大气主 要成分为氧原子, 本文取 $\alpha_{E}=0.93$ (Doornbos等, 2009).

阻尼系数主要与温度相关, 有效面积主要与卫星 姿态相关, 因此这两个参数随卫星运动而变化. 图 1 为 2009年第32天CHAMP卫星阻尼系数和有效面积的乘 积随时间的变化. 可以看出, 该乘积最大可以达到约 2.35 , 而最小值只有约 1.95 , 因此相对变化可达到 $20 \%$.

\section{2 最小二乘法计算加速度计数据标定因子}

如 2.3 节所述, 使用CHAMP卫星加速度计数据反 


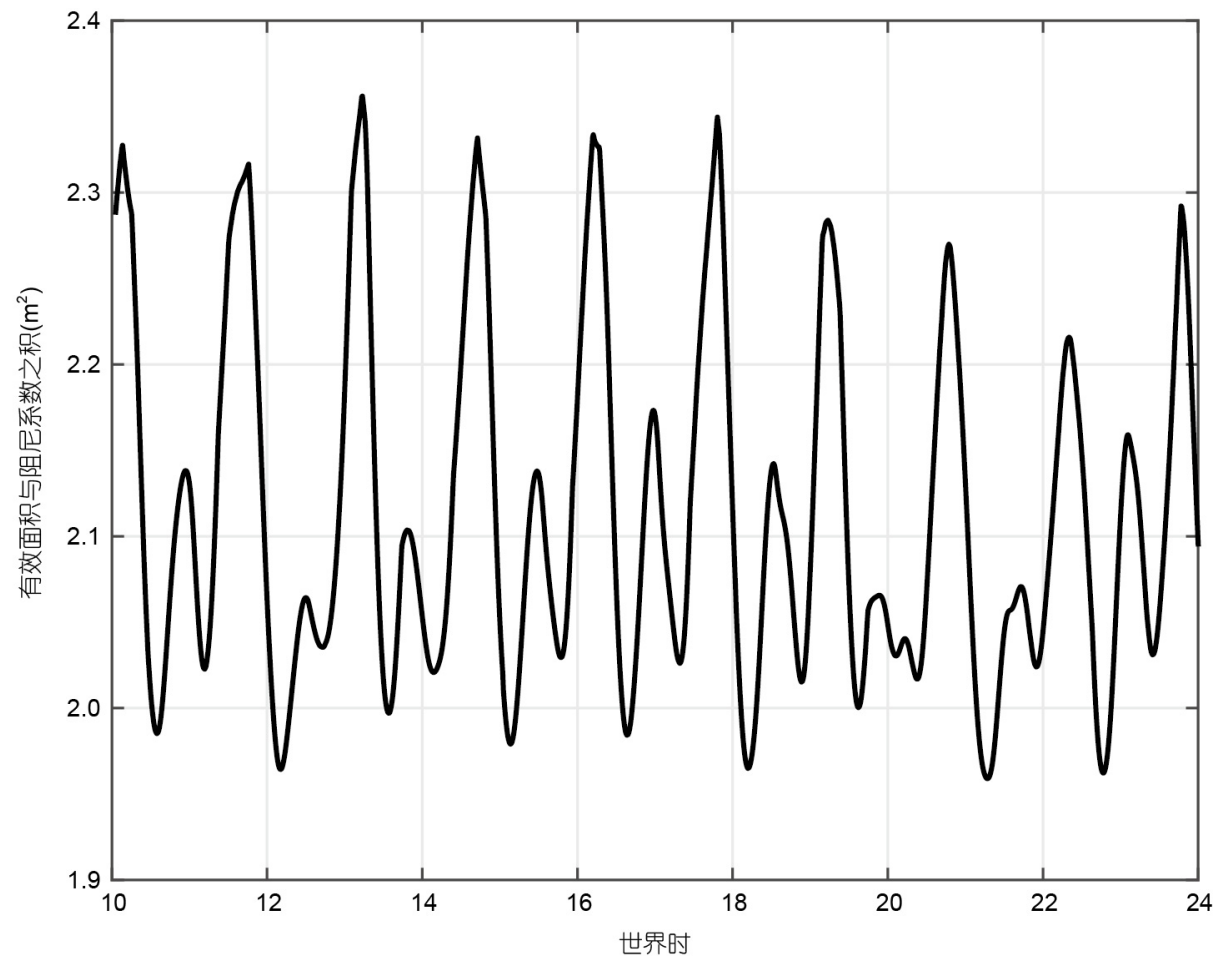

图 12009 年第 32 天大气阻尼系数与有效面积乘积的变化

演热层大气密度需要先对加速度计数据进行标定, 如 式(17)所示, 计算尺度标定因子Scale和偏差标定因子 Bias. 本文将使用能量衰减法对加速度计数据进行标 定. 沿卫星运动方向, 非保守力主要为大气拖曳力, 用 式(17)替换式(11)中非保守力(徐天河和杨元喜, 2004), 有

$$
\begin{aligned}
& \int\left(\text { Scale } \times a_{\text {observe }}+\text { Bias }\right) \cdot|v| \mathrm{d} t \\
= & \left(\frac{1}{2}|v|^{2}-V_{\text {SUN }}-V_{\text {MOON }}\right. \\
& \left.-\frac{1}{2} \omega^{2}\left(x^{2}-y^{2}\right)-V-E_{0}\right)\left.\right|_{t_{0}} ^{t_{1}},
\end{aligned}
$$

式中, $|v|$ 代表卫星运动速度的模. 采用与式(12)相似的 处理方法, 得到

$$
\begin{aligned}
& \text { Scale } \times a_{\text {observe }}+\text { Bias } \\
= & \left(\frac{1}{2}|\nu|^{2}-V_{\text {SUN }}-V_{\text {MooN }}\right. \\
& \left.-\frac{1}{2} \omega^{2}\left(x^{2}-y^{2}\right)-V-E_{0}\right)\left.\right|_{t_{0}} ^{t_{1}} /|v| \Delta t .
\end{aligned}
$$

将上式右边第 $i$ 个数据点记为 $A^{i}$, 对于第 $i$ 个观测量 $a_{\mathrm{observe}}^{i}$, 可得到

$$
\text { Scale } \times a_{\text {observe }}^{i}+\text { Bias }=A^{i} .
$$

利用最小二乘法估计, 以一天作为拟合弧段, 可 以计算出加速度计数据尺度和偏差标定因子. 根据 Doornbos等(2009), 我们固定尺度因子Scale为GFZ官方 参考值 0.8333 , 使用最小二乘法只解算偏差因子 Bias. 图2为2007 2009年期间解算出的加速度计数据每天 的偏差标定因子Bias, 红线代表 20 天滑动平均值. 接 着, 根据式(19)计算大气密度. 如图3所示, 使用加速度 计数据反演的大气密度与美国科罗拉多大学团队反 演结果(Sutton等, 2007)对比具有较好的一致性, 相关 性系数高达 0.99 以上. 能量衰减法标定加速度计数据 在计算上更加便捷、高效.

\section{4 反演密度结果比较及讨论}

设置时间间隔 $\Delta t=150 \mathrm{~s}$, 考虑阻尼系数和有效面积 随时间的变化, 以2009年第32天为例, 分别使用半长轴 衰减法和能量衰减法反演热层大气密度. 结果如图 4 


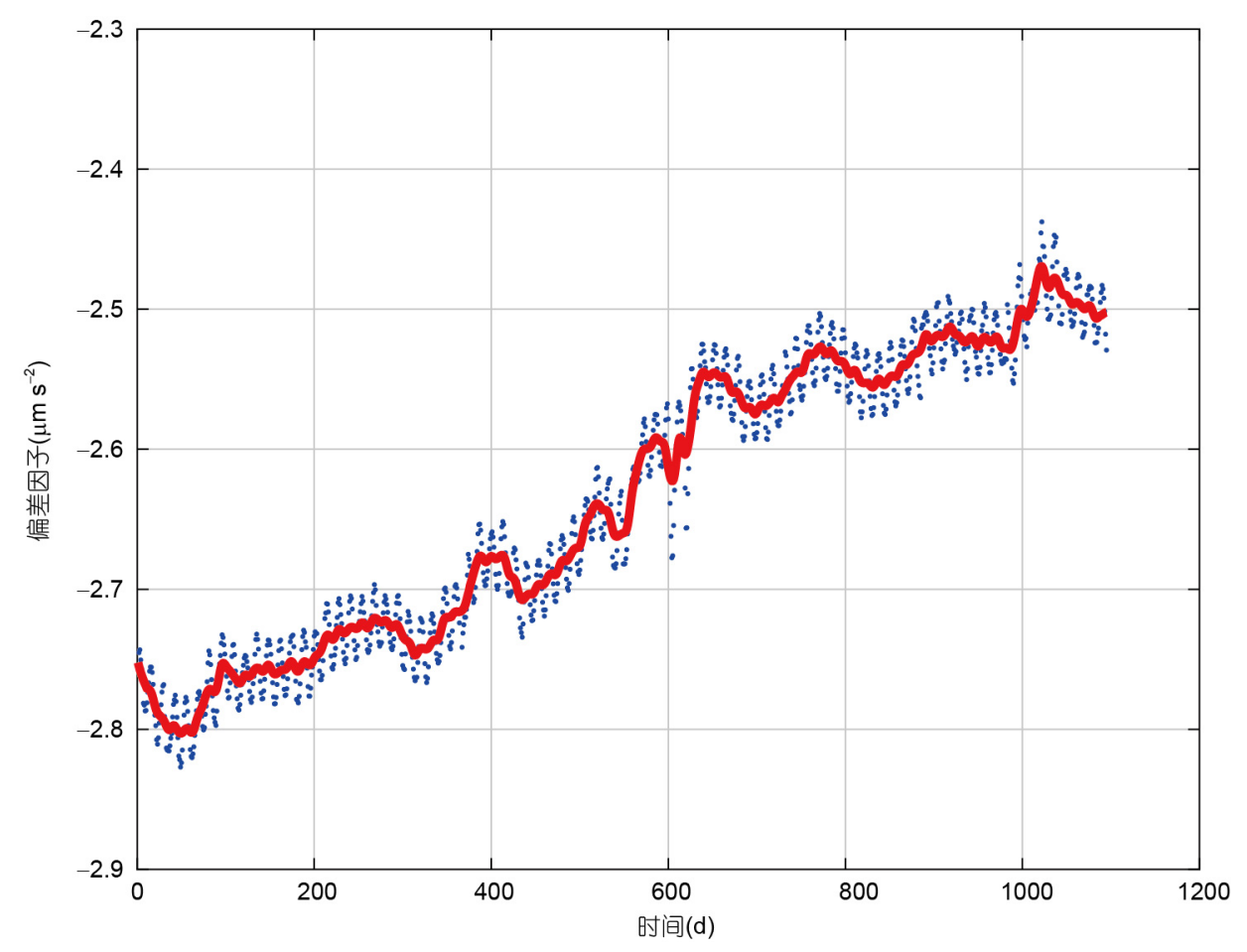

图 2 固定尺度因子计算2007 2009年期间偏差因子 红线为 20 天滑动平均值

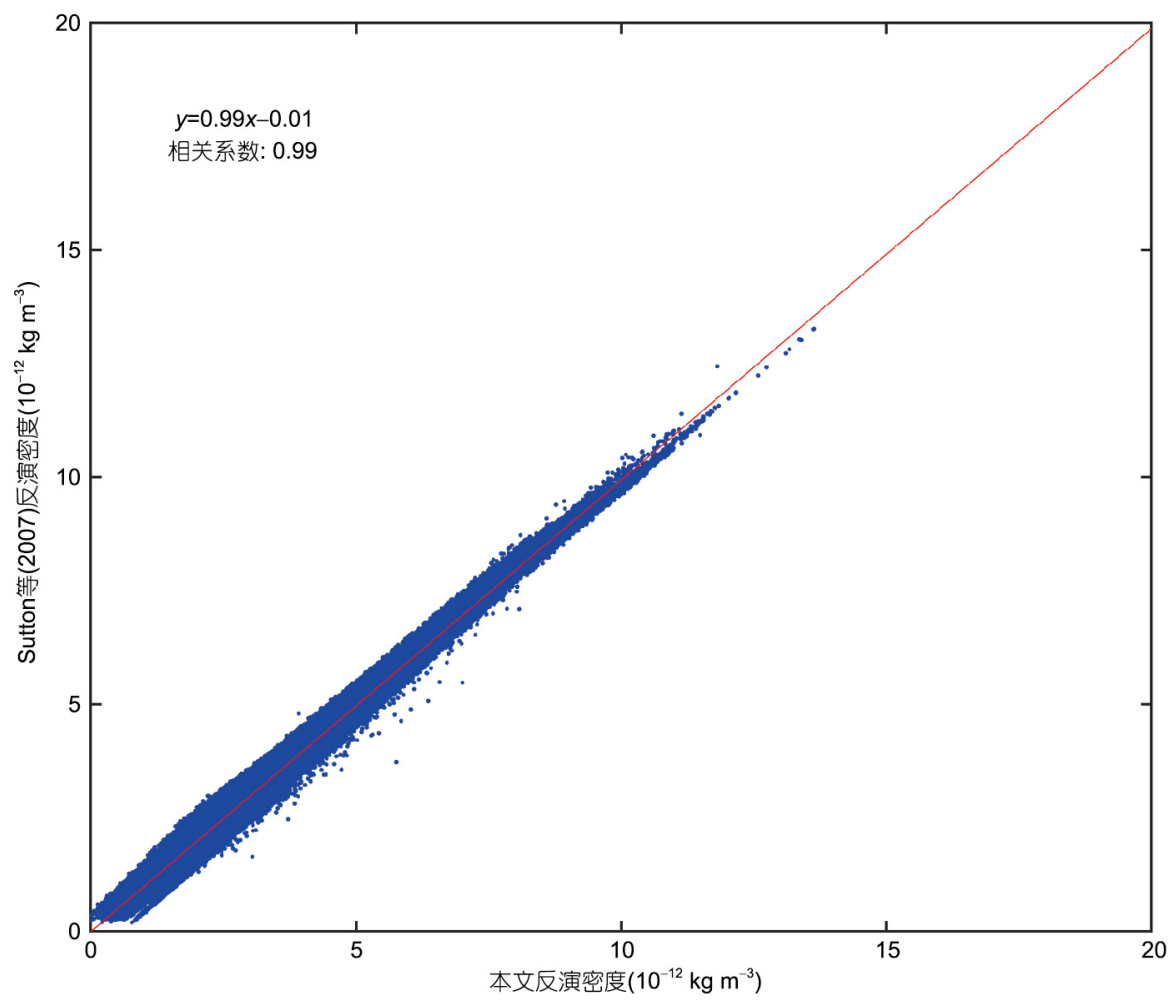

图 3 本文使用加速度计数据反演密度与Sutton等(2007)结果对比 


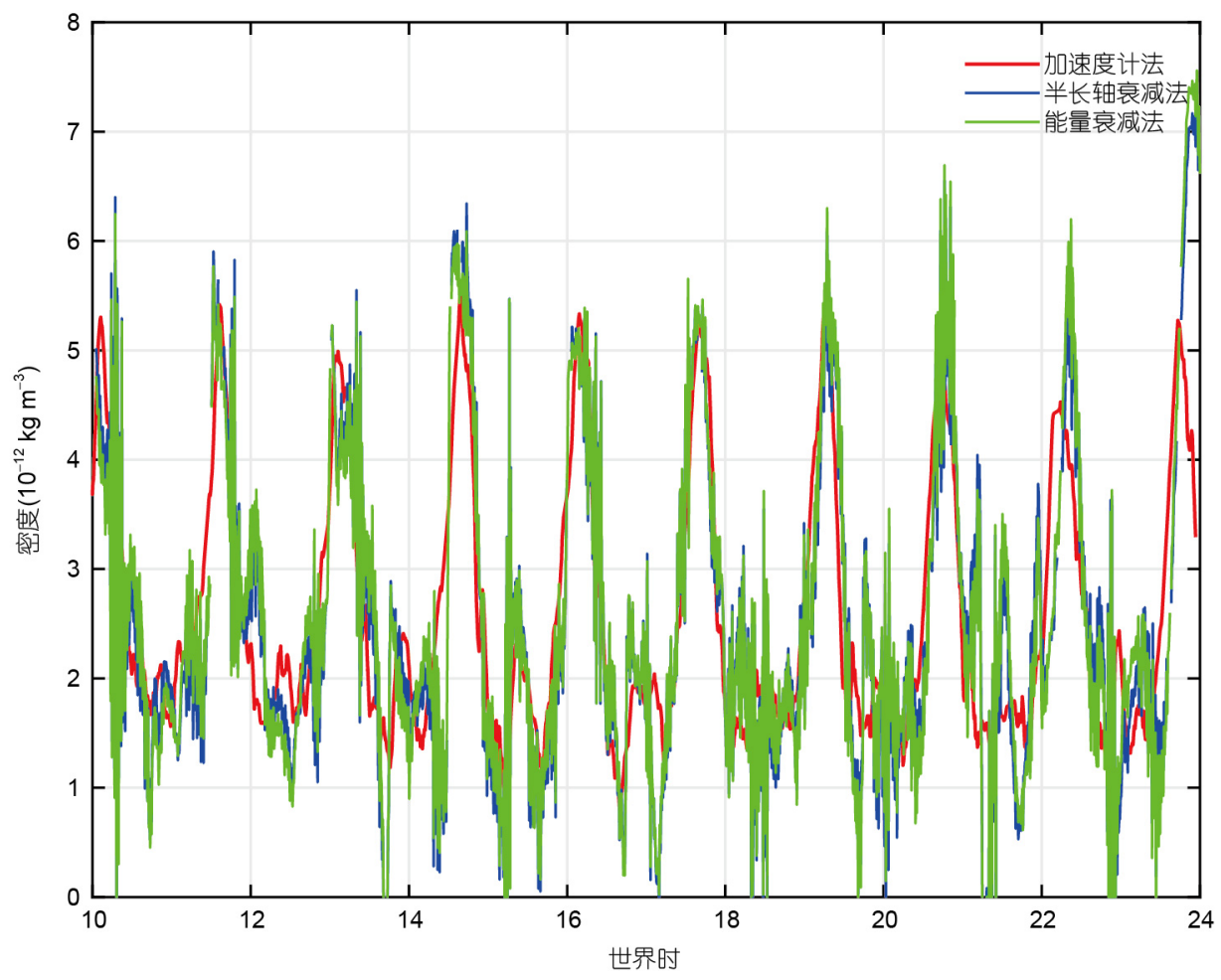

图 4 2009年第32天半长轴衰减法反演密度(蓝色)、能量衰减法反演密度(绿色)与加速度计反演密度(红色)对比 积分时间为 $2.5 \mathrm{~min}$

所示, 半长轴衰减法和能量衰减法所得结果一致, 符 合 2.2 节理论推导结果. 使用能量衰减法反演热层大 气密度, 不需要进行轨道外推, 运算速度相对于半长 轴衰减法提升约一个数量级, 而且计算精度不会下降. 因此在下面的对比过程中, 使用精密轨道数据反演大 气密度均采用能量衰减法. 这里需要说明的是时间间 隔为 $150 \mathrm{~s}$ 时, 半长轴的平均衰减量约为 $0.2 \mathrm{~m}$ 与 $\Delta a$ 的不 确定度大小相当. 反演结果显示, 在RSO数据的定轨 精度下, 选择 $150 \mathrm{~s}$ 的时间间隔基本可以反演出大气密 度, 但是反演结果涨落较大, 其主要原因是定轨误差 造成的噪声.

图5给出了使用精密轨道数据和加速度计数据反 演2009年2月 4 5 日、 2009 年 2 月 24 日以及 2009 年 3 月 21 日的热层大气密度. 需要指出的是, 选择这几天结 果比较的原因是Sang等(2012)也比较了该时段精密轨 道与加速度计数据反演的大气密度. 两种方法所得密 度结果比较吻合, 这个结论与Sang 等(2012)结果是一 致的, 这也验证了我们所采用的反演方法的正确性. CHAMP卫星每天都会多次调姿, 调姿过程姿态控制
火箭启动, 因此, 调姿前后反演结果不可信, 故将其去 除. 因此, 精密轨道数据反演密度不连续部分是由于 卫星调姿, 加速度计数据反演密度不连续部分是由于 姿态数据缺失.

无论半长轴衰减法还是能量衰减法反演的密度 均和加速度计数据反演结果基本吻合, 但是夜侧大气 密度较小, 半长轴和能量均衰减较小, 导致计算结果 不稳定, 误差较大. 比如图 4 和 5 中出现小于零以及高 频跳变结果是不合理的. 增加时间步长, 可以有效提 升计算的稳定性. 如图6所示, 将图4所示2009年第32 天密度反演过程中的时间步长增加到 $20 \mathrm{~min}$, 精密轨 道数据反演密度与加速度计数据反演密度基本一致. 需要指出的是夜侧半长轴衰减量更小, 以 $150 \mathrm{~s}$ 的时间 间隔为例, 半长轴衰减量约为 $0.1 \mathrm{~m}$, 反演噪声更大. 图 6 与 4 相比, 时间间隔增加为 $1200 \mathrm{~s}$, 这使得夜侧小周期 的密度变化全部被平滑. 实际上, 如图4所示, 精密轨 道数据反演密度可以观察到与加速度计数据反演密 度相似的夜间小周期密度变化特征. 因此, 长时间间 隔平滑噪声的同时, 夜间密度的小尺度变化特征也被 

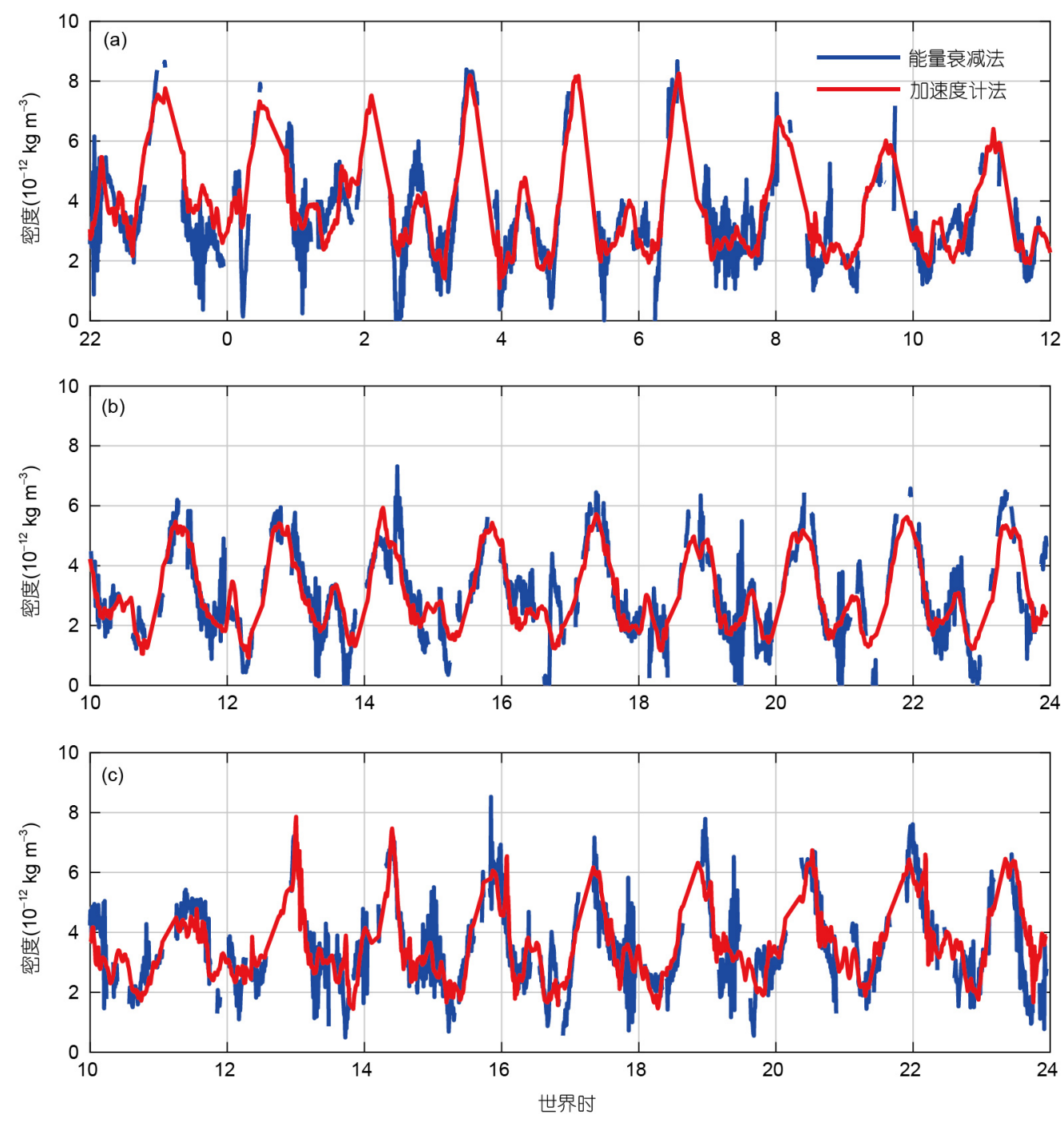

图 52009 年2月4 5 日(a)、2月 24 日(b)、3 月 21 日(c)精密轨道反演密度与加速度计反演密度对比

平滑掉.

下面使用精密轨道数据基于能量法反演2007 2009 年期间的密度结果, 并与加速度计数据反演结果对比, 研究精密轨道数据反演密度方法的适用条件. 图7a、 $\mathrm{d}$ 和 $\mathrm{g}$ 分别代表2007 2009年期间使用精密轨道数据反 演密度和使用加速度计数据反演密度的相关系数与 时间间隔之间的关系. 其中, 蓝色线条代表以一天作 为一个弧段计算的相关系数, 然后取全年平均, 红色线 条代表以一年作为弧段计算的相关系数. 图7b、e 和h 分别代表2007 2009年以 20min作为时间间隔, 使用精 密轨道数据反演密度和使用加速度计数据反演密度 之比的统计直方图, 并对其正态拟合. 图7c、f和 $\mathrm{i}$ 分别
代表2007 2009年以20min作为时间间隔, 使用精密轨 道数据反演密度和使用加速度计数据反演密度的分 布图. 需要说明的是, 在低密度段加速计数据反演密 度比精密轨道数据反演密度略小的原因与加速度计 数据标定误差有关. 卫星运行地方时为 $6 \sim 18 \mathrm{LT}$ 时, 大 气密度相对较小, 从而在该区间加速度计数据标定因 子计算误差较大. 根据图7的结果, 我们可以得出以下 结论: 增加时间间隔可以提升计算结果的稳定性, 平 滑掉高频部分, 但是可能会抹去热层大气的一些快速 变化特征, 因此时间间隔最好不超过 $25 \mathrm{~min}$. 设置时间 间隔为 $20 \mathrm{~min}$, 精密轨道数据反演密度和加速度计数据 反演密度之比统计结果以及密度分布表明, 两种方法 


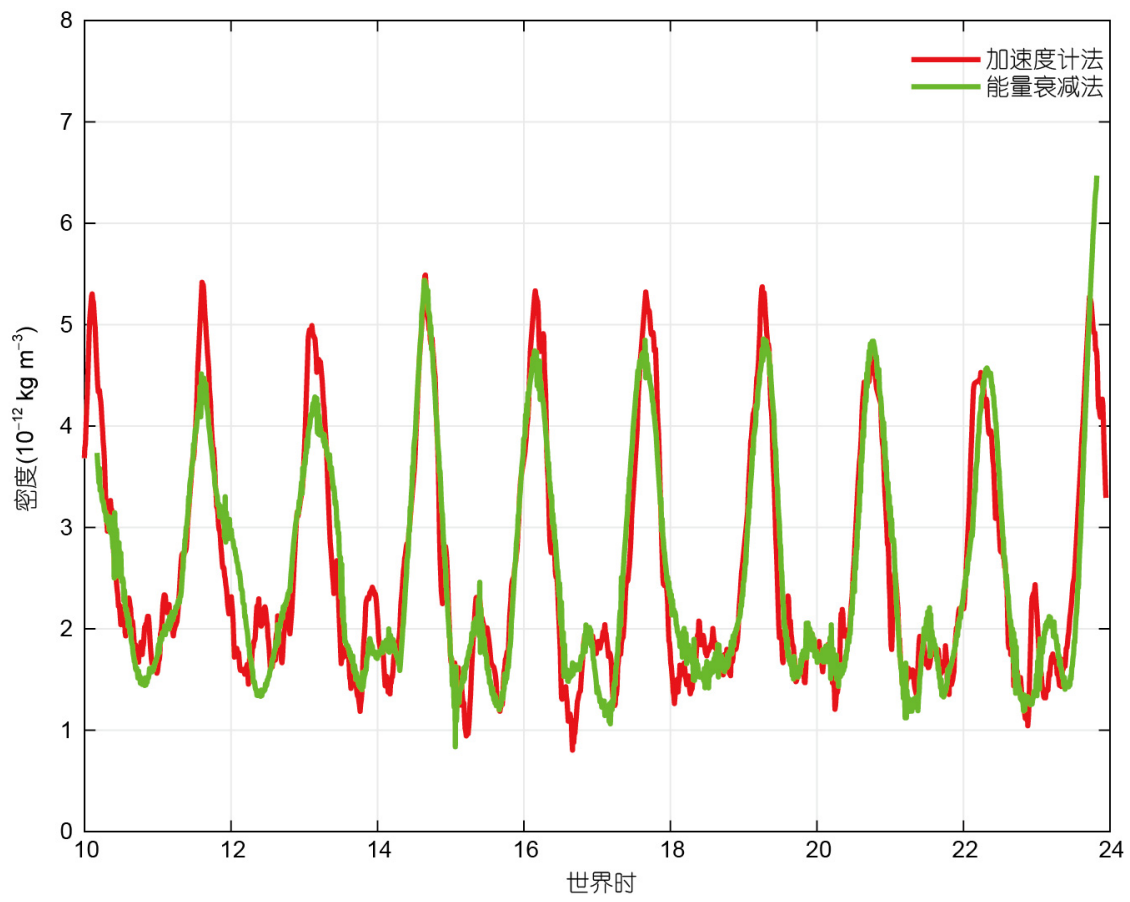

图 62009 年第32天精密轨道反演密度(绿色)与加速度计反演密度(红色)对比 积分时间 $20 \mathrm{~min}$
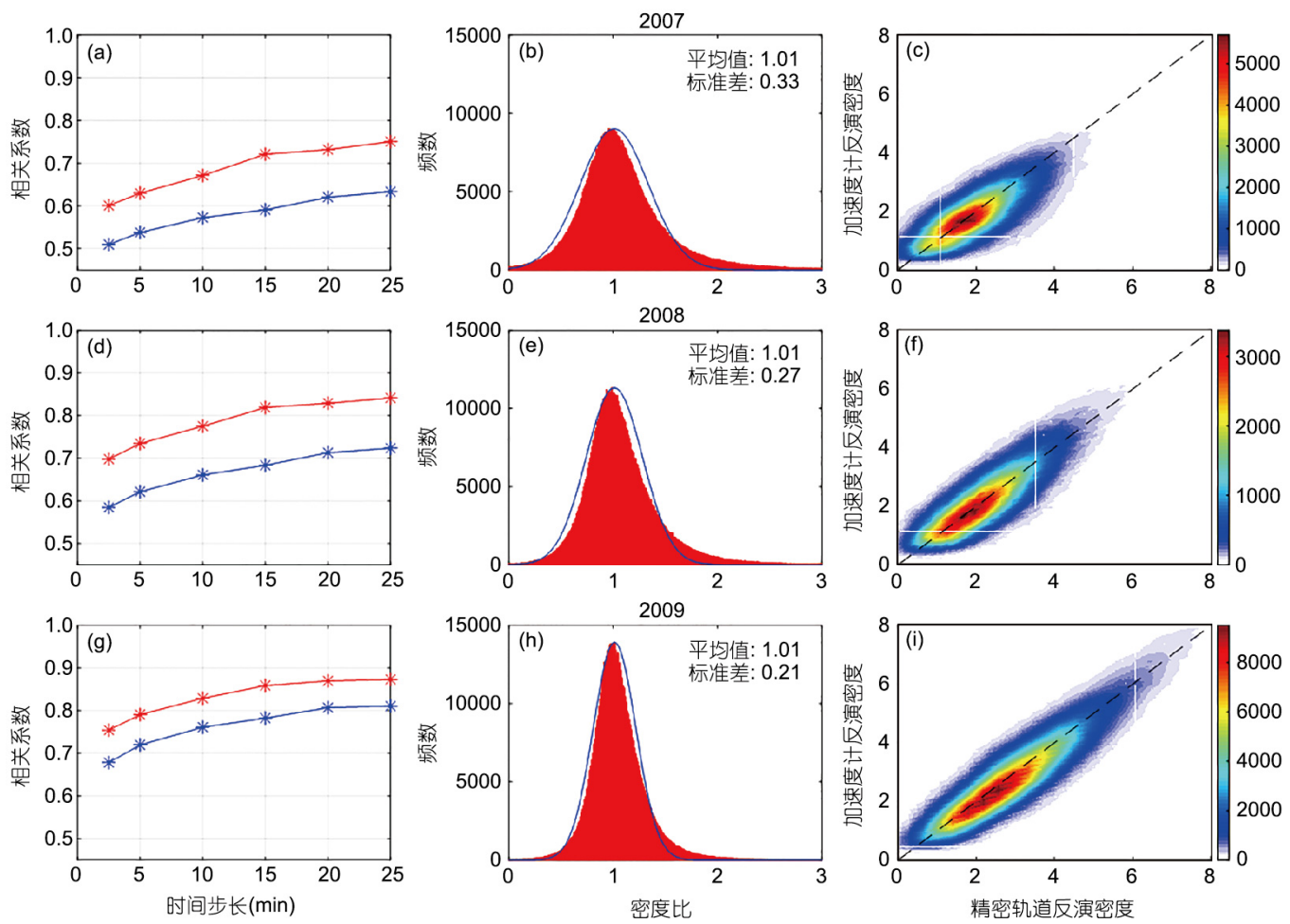

图 7 精密轨道反演密度与加速度计反演密度的相关系数与时间步长关系、时间步长为 $20 \mathrm{~min}$ 情况下精密轨道反演 密度与加速度计反演密度之比频数统计以及密度分布

(a) (c)、(d) (f)、(g) (i) 分别代表2007 2009年相关系数与时间步长关系、密度之比频数统计以及密度分布; 密度单位: $10^{-12} \mathrm{~kg} \mathrm{~m}^{-3}$ 
所得密度没有系统性偏差, 标准差较小, 分别为 0.33 、 0.27 和 0.21 , 且逐年下降, 这是因为卫星轨道高度下降, 大气密度增加, 使得反演精度提升.

图8给出精密轨道数据、加速度计数据反演的热 层大气密度与MSIS00对比情况. 可以得出如下结论: (1) 精密轨道数据反演密度、加速度计数据反演密度 和MSIS00相比, 存在约 $25 \%$ 的系统偏差; (2) 精密轨道 数据反演密度与加速度计数据反演密度没有明显系 统偏差, 这是由于加速度计数据偏差因子是通过精密 轨道数据标定而来, 但标准差偏大.

使用能量衰减法反演热层大气密度以及计算加 速度计数据标定系数均依赖于单位时间能量的衰减, 因此衰减量越大, 计算精度越高. 另外, 需要指出的是, 反演大气密度的精度不仅与高度相关, 而且与地方时 相关. 图9以 2009 年上升轨道为例, 展示了反演结果相
关性与卫星地方时的关系. 卫星日侧运行时, 比如地 方时 12 点左右, 精密轨道数据反演密度和加速度计数 据反演密度相关性较高, 同时加速度计数据反演密度 和MSIS00相关性也较高, 而地方时 24 点左右, 相关性 则较小. 这是由于日侧太阳辐射强, 密度较高, 卫星运 行过程中单位时间能量衰减较多, 反演精度也较高.

利用精密轨道数据反演密度结果可用以研究磁 暴对热层密度的影响. 如图10所示, 以2008年上升轨 道为例, 取密度的轨道平均, 其中蓝色线条代表精密 轨道数据反演密度, 红色线条代表加速度计数据反演 密度; 以Dst指数表征地磁活动. 可以看出, 精密轨道 数据反演密度与加速度计数据反演密度较为一致. 除 了卫星地方时和季节引起的变化外, 热层密度还呈现 明显的数天周期性变化, 并与Dst指数变化一致. Lei等 (2011b) 已阐明该时间段观测到热层大气周期变化与
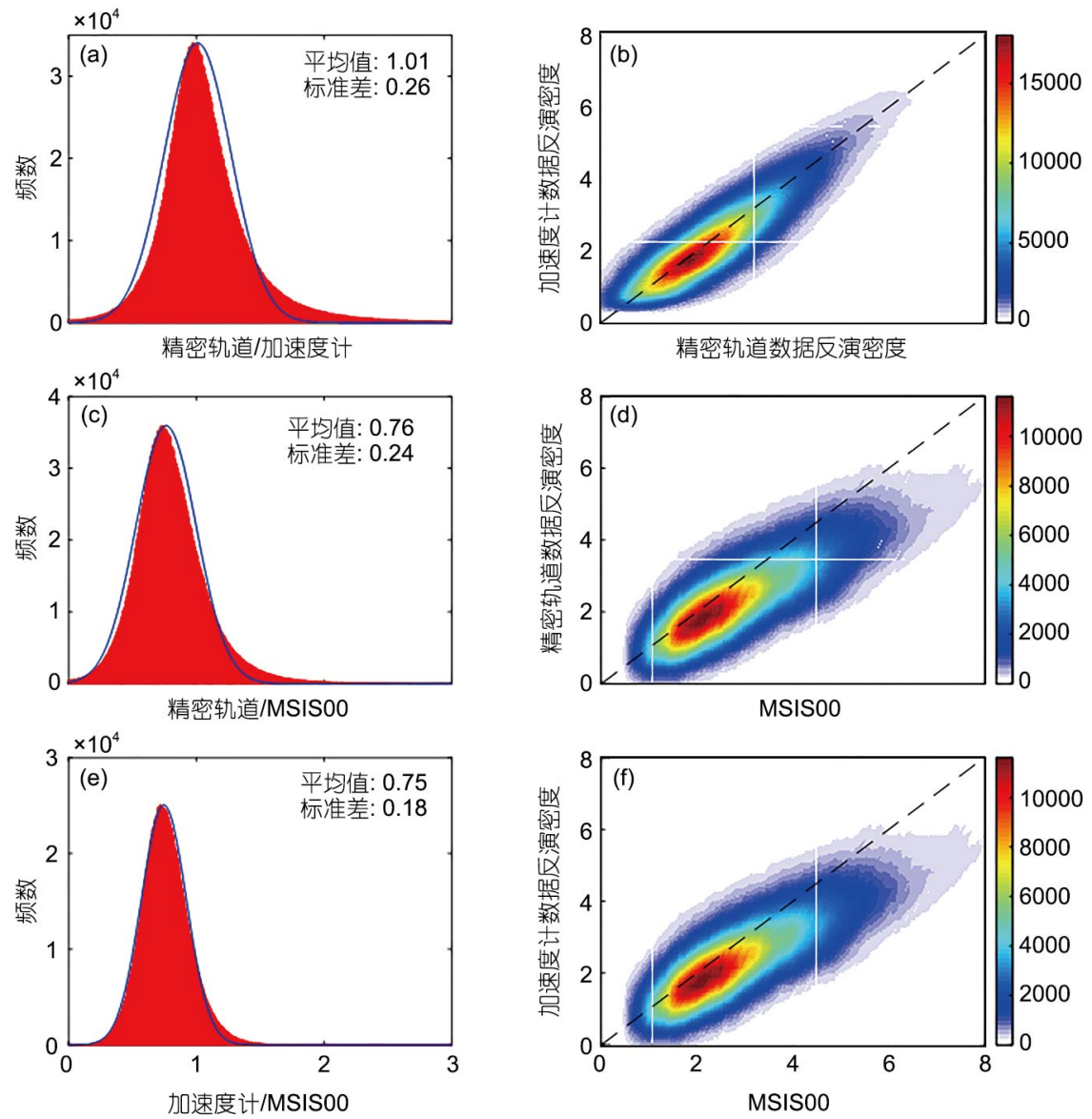

图 82007 2009年精密轨道反演密度与加速度计反演密度之比频数统计以及密度分布((a)和(b))、精密轨道反演密度与 MISIS00密度之比频数统计以及密度分布((c)和 (d)), 加速度计反演密度与MISISO0 密度之比频数统计以及密度分布 $((\mathrm{e})$ 和(f)) 密度单位为 $10^{-12} \mathrm{~kg} \mathrm{~m}^{-3}$ 

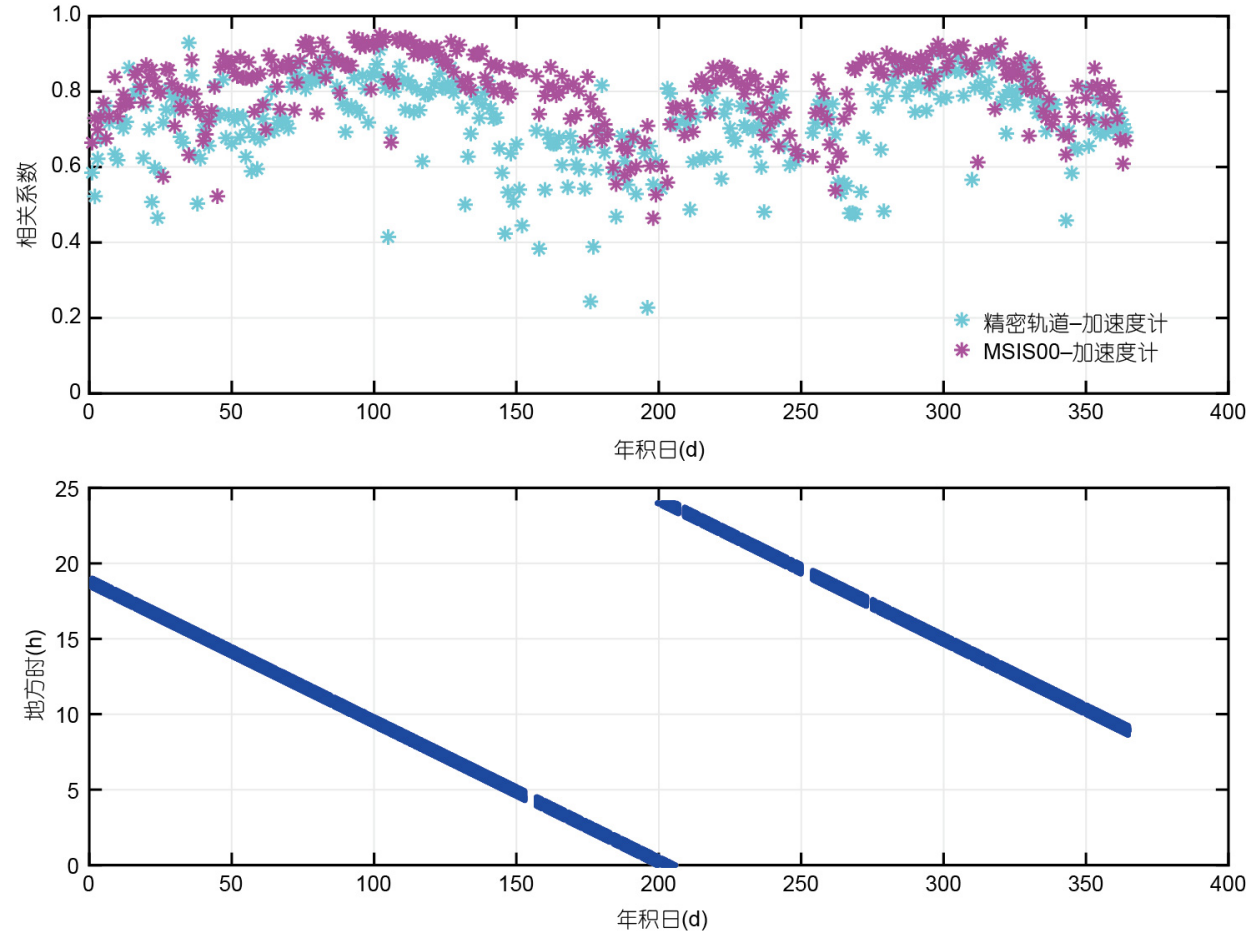

图 92009 年上升轨道精密轨道反演密度与加速度计反演密度的相关系数以及加速度计反演密度与MSISO0的相关系 数变化情况

下图给出卫星过赤道的地方时
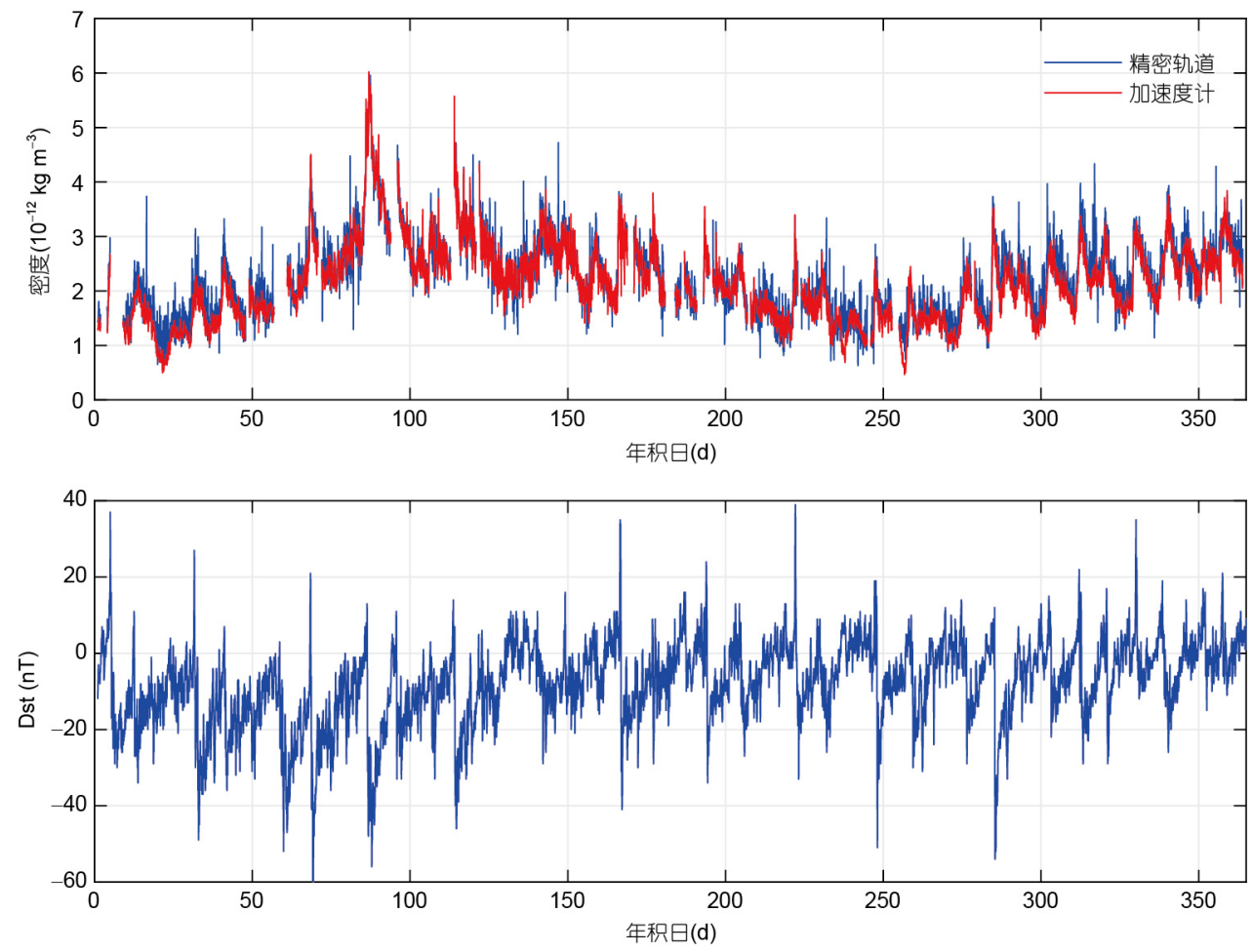

图 102008 年上升轨道平均密度与Dst指数变化 
冕洞引起的太阳风高速流导致地磁活动相关.

\section{5 总结}

本文比较了利用CHAMP卫星精密轨道数据以及 加速度计观测数据反演热层大气密度两种方法, 重点 分析了能量衰减法反演大气密度的方法, 同时将反演 结果与经验模式结果对比, 并统计分析了 2007 2009 年期间的反演结果. 我们得出如下结论:

(1) 半长轴衰减法和能量衰减法反演得到的热层 大气密度是一致的, 但能量衰减法计算速度较半长轴 衰减法大幅提高.

(2) 反演密度的时间间隔较短时可能由于大气密 度累积效应不够, 反演噪声较大. 我们分析显示对于 CHAMP卫星, 采用 $20 \mathrm{~min}$ 左右的积分时间较为合适.

(3) 应用能量衰减法反演密度的同时, 可对加速度 计数据进行标定. 能量衰减法所计算出的加速度计数 据偏差因子具有较高准确性, 用其标定后的加速度计 数据可较为准确的反演热层大气密度.

(4) 使用精密轨道数据反演密度的计算精度与卫 星高度、地方时等相关. 日侧正午计算结果精度较高, 夜侧以及晨昏线附近反演精度较低.

致谢感谢Sutton博士提供的数据和帮助, 以及唐歌实 研究员的有益讨论.

\section{参考文献}

李济生. 1995. 人造卫星精密轨道确定. 北京: 解放军出版社. 100-120 李文文, 李敏, 施闯, 赵齐乐. 2016. 基于GRACE星载加速度计数据 的热层密度反演. 地球物理学报, 59: 3159-3174

刘林. 1992. 人造地球卫星轨道力学. 北京: 高等教育出版社. 84-99, 389-408

熊永清, 汪宏波, 赵长印, 许晓丽. 2011. CHAMP加速仪资料的快速 校标研究. 天文学进展, 29: 228-237

徐天河, 杨元喜. 2004. 利用现有重力场模型求定CHAMP卫星加速 度计修正参数. 测绘学报, 33: 200-204

Berger C, Biancale R, Barlier F, Ill M. 1998. Improvement of the empirical thermospheric model DTM: DTM94-A comparative review of various temporal variations and prospects in space geodesy applications. J Geodesy, 72: 161-178

Bowman B R, Tobiska W K, Marcos F A, Huang C, Lin C, Burke W. 2008. A new empirical thermospheric density model JB2008 using new solar and geomagnetic indices. In: AIAA/AAS Astrodynamics
Specialist Conference, and Exhibit, Guidance, Navigation, and Control and Co-located Conferences. Honolulu. 6438

Bruinsma S, Biancale R. 2003. Total density retrieval with STAR. In: First CHAMP Mission Results for Gravity, Magnetic and Atmospheric Studies. Berlin: Springer. 193-199

Bruinsma S, Tamagnan D, Biancale R. 2004. Atmospheric densities derived from CHAMP/STAR accelerometer observations. Planet Space Sci, 52: 297-312

Calabia A, Jin S. 2016. Assessment of conservative force models from GRACE accelerometers and precise orbit determination. Aerospace Sci Tech, 49: 80-87

Doornbos E, Förster M, Fritsche B, van Helleputte T, van den Ijssel J, Koppenwallner G, Lühr H, Rees D, Visser P, Kern M. 2009. Air density models derived from multi-satellite drag observations. In: Proceedings of ESAs Second Swarm International Science Meeting. Potsdam. 24-26

Doornbos E. 2012. Thermospheric Density and Wind Determination from Satellite Dynamics. Berlin: Springer Science \& Business Media

Emmert J T. 2015. Thermospheric mass density: A review. Adv Space Res, 56: 773-824

Flury J, Bettadpur S, Tapley B D. 2008. Precise accelerometry onboard the GRACE gravity field satellite mission. Adv Space Res, 42: 1414-1423

Förste C, Schwintzer P, Reigber C. 2002. Format description: The CHAMP data format. CH-GFZ-FD-001

Hedin A E. 1987. MSIS-86 thermospheric model. J Geophys Res, 92: $4649-4662$

Jekeli C. 1999. The determination of gravitational potential differences from satellite-to-satellite tracking. Celestial Mech Dynamical Astron, 75: $85-101$

König R, Schwintzer P, Reigber C. 2001. Format description: The CHAMP data format. CH-GFZ-FD-002

Lei J, Thayer J P, Lu G, Burns A G, Wang W, Sutton E K, Emery B A. 2011a. Rapid recovery of thermosphere density during the October 2003 geomagnetic storms. J Geophys Res, 116: A03306

Lei J, Thayer J P, Wang W, McPherron R L. 2011b. Impact of CIR storms on thermosphere density variability during the Solar minimum of 2008. Sol Phys, 274: 427-437

Lei J, Burns A G, Thayer J P, Wang W, Mlynczak M G, Hunt L A, Dou X, Sutton E. 2012. Overcooling in the upper thermosphere during the recovery phase of the 2003 October storms. J Geophys Res, 117: A03314

Lühr H, Grunwaldt L, Förste C, Schwintzer P, Reigber C. 2001. CHAMP reference systems, transformations and standards. Internal publication. GFZ Potsdam. CH-GFZ-RS-002

Marcos F A. 1990. Accuracy of atmospheric drag models at low satellite 
altitudes. Adv Space Res, 10: 417-422

Michalak G, Baustert G, König R, Reigber C. 2003. CHAMP Rapid Science Orbit Determination-Status and Future Prospects. In: First CHAMP Mission Results for Gravity, Magnetic and Atmospheric Studies. Berlin: Springer. 98-103

Pavlis N K, Holmes S A, Kenyon S C, Factor J K. 2008. An earth gravitational model to degree 2160: EGM2008. EGU General Assembly. $13-18$

Petit G, Luzum B. 2010. IERS conventions (2010). IERS Technical Note 36. Frankfurt am Main: Verlag des Bundesamts für Kartographie und Geodäsie

Picone J M, Emmert J T, Lean J L. 2005. Thermospheric densities derived from spacecraft orbits: Accurate processing of two-line element sets. J Geophys Res, 110: A03301

Picone J M, Hedin A E, Drob D P, Aikin A C. 2002. NRLMSISE-00 empirical model of the atmosphere: Statistical comparisons and sci- entific issues. J Geophys Res, 107: SIA 15-1-SIA 15-16

Reigber C, Lühr H, Schwintzer P. 2002. CHAMP mission status. Adv Space Res, 30: 129-134

Sang J, Smith C, Zhang K. 2012. Towards accurate atmospheric mass density determination using precise positional information of space objects. Adv Space Res, 49: 1088-1096

Sentman L H. 1961. Free molecule flow theory and its application to the determination of aerodynamic forces. Sunnyvale: Lockheed Missiles And Space Co Inc

Sutton E K, Nerem R S, Forbes J M. 2007. Density and winds in the thermosphere deduced from accelerometer data. J Spacecr Rockets, 44: $1210-1219$

Tapley B D, Bettadpur S, Watkins M, Reigber C. 2004. The gravity recovery and climate experiment: Mission overview and early results. Geophys Res Lett, 31: L09607 